\title{
Material Parameters Acquisition and Sound Insulation Performance analysis of Membrane-type Acoustic Metamaterials Applied for Transformer
}

\author{
Ma Yuchao ${ }^{1 *}$, Mo Juan ${ }^{1}, \mathrm{Xu} \mathrm{Ke}^{2}$, Li Xiang ${ }^{1}$, Sun Xinbo ${ }^{3}$ \\ ${ }^{1}$ China Electric Power Research Institute, Beijing 100055, China \\ ${ }^{2}$ Heze Power Supply Company, Shandong Electric Power Corporation, Heze, 274000, China \\ ${ }^{3}$ School of Mechanical Engineering, Xi' an Jiaotong University, Xi'an, 710049, China
}

\begin{abstract}
As a light-weight and ultra-thin artificial material, acoustic metamaterial have more different attributes than natural material. The study of sound insulation for acoustic metamaterial is hot, and the membrane-type acoustic metamaterials supplement the deficiency of linear sound insulation materials. The physical material parameters (young modulus and loss factors)of base material of membrane-type acoustic metamaterials (PVC) is obtained by cantilever beam dynamic measurement method. The acoustic metamaterial sound insulation analysis is simulated by CAE method based on the material parameters that measured. The configuration of the simulation accuracy is measured on impedance tube, and the design work of the acoustic metamaterial sound insulation for transformer is provided. The relationship between sound insulation and the mass on membrane-type acoustic metamaterial at the different frequencies $(100 \mathrm{~Hz}$ to $500 \mathrm{~Hz}$ ) provides the reference to set sound insulation frequency.
\end{abstract}

\section{Introduction}

Metamaterial has become a hot research topic in international academic circles in recent years. New ideas, new achievements and new application fields of metamaterials emerge one after another, making the research field of metamaterials develop rapidly. Metamaterial is a kind of synthetic composite-structure or composite-material thin-film acoustic metamaterial which surpasses the physical properties of existing materials in nature and has some peculiar physical properties. The metamaterial is formed by fixing the elastic thin film of the additional vibrator on the support frame. Due to the great difference in the density of the vibrator and the thin film, under the excitation of sound waves of a specific frequency, the vibration around the vibrator is inverted and a sound insulation frequency band appears. The outstanding advantage of thin-film acoustic metamaterial is that it can effectively isolate low-frequency noise under the condition of ensuring light weight. Systematic and in-depth research on thin-film acoustic metamaterials can provide a solid theoretical basis for its engineering applications.

Liu et al. [1] designed the first prototype of acoustic metamaterial structure by arranging the lead ball coated with viscoelastic soft material cyclically. For two-dimensional structures, Shen Ping and Mei Jun from Hong Kong University of Science and Technology have acquired the analytical expressions of equivalent elastic modulus and mass density of metamaterials with cylindrical structural elements arranged in the fluid under the condition of long wave using multi-scattering analysis of energy band structure [2]. Thin-film acoustic metamaterial is a kind of light low-frequency sound insulation material, which was proposed by Hong Kong University of Science and Technology [3]. They fixed the elastic thin film with additional mass on a relatively hard support frame to realize effective sound insulation around any specific frequency in the frequency range of $50 \sim 1000 \mathrm{~Hz}$. In 2010, Naify et al. studied the influence of additional weight mass and film tension on the sound insulation range of thin-film acoustic metamaterial by impedance tube test and finite element simulation, and carried out a series of dynamic analysis on the resonance frequency and film vibration at the peak of sound insulation using laser vibration testing instruments [4]. From the research progress of acoustic metamaterials [5-8], we find that the focus of research on acoustic metamaterials is the effective parameters of materials, and the parameters directly affect the sound insulation effect [9-12]. In this paper, we mainly study the sound insulation characteristics of thin-film acoustic metamaterial composed of rigid frame, flexible film and additional mass. Firstly, the acoustic band gap of metamaterial is studied by using phononic crystal band gap theory, the parameters of elastic film are acquired by using experiment table, and the relationship between the sound insulation of metamaterial and its band gap frequency interval is acquired by finite element simulation. Then, the sound insulation characteristics of

\footnotetext{
*Corresponding author's e-mail: myc2011bjut@163.com
} 
thin-film metamaterial cells are studied by finite element simulation. Finally, the validity of the simulation results is verified by standing wave tube experiments.

\section{Mechanic Model of Acoustic Metamaterial}

When the film is disturbed by external force, the force to restore it to its equilibrium state is mainly the tension. The elastic stiffness of the material itself is negligible compared to tension. As shown in Fig. 1, the force on the panel is analysed and its vibration equation is summarized.

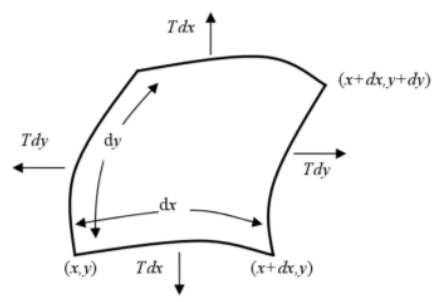

Fig. 1 Schematic Diagram of Stress in Case of Lateral Vibration of Membrane Surface Element

Under the condition of cylindrical coordinates, the stress can be written as the following

$$
T\left(\frac{\partial^{2}}{\partial r^{2}}+\frac{1}{r} \frac{\partial}{\partial r}+\frac{1}{r^{2}} \frac{\partial^{2}}{\partial \theta^{2}}\right)=\rho_{s}\left(\frac{\partial^{2} w}{\partial t^{2}}\right)
$$

The membrane structure vibrates under the action of incident sound pressure, and the membrane structure vibrates to generate reradiated sound pressure. Here, the radiated sound pressure at the transmission end is referred to as the transmitted sound pressure, and the transmitted sound pressure is acquired by Rayleigh integration, which shows as Eq2 and Eq3.

$$
p_{t}(r, \theta, \phi, t)=\frac{j \omega \rho_{a} e^{j \omega t}}{2 \pi} \int_{0}^{L_{x}} T \int_{0}^{L_{y}} \tilde{v}(x, y) \frac{e^{-j k_{a} R}}{R} d x d y
$$

$$
R=r \sqrt{1-\frac{2 \sin \theta \cos \phi}{r} x+\left(\frac{x}{r}\right)^{2}-\frac{2 \sin \theta \cos \phi}{r} y+\left(\frac{y}{r}\right)^{2}}
$$

However, it is difficult to acquire the solution of the above equation by analytical method, so most of them are acquired based on finite element method.

\section{Acquiring the Material Parameters of Membrane Materials}

In order to be able to accurately CAE the simulation results, the properties of experimental materials in the simulation process must be accurate. For most membranes, it is difficult to acquire the mechanical properties of membrane materials by conventional methods due to the uncertainty of materials. At present, there is a method for measuring material properties by vibration method. In the form of experimental splines, the Young's modulus and loss factor of the material can be conveniently acquired on the damping experimental table through a force hammer and an accelerometer, which is very convenient for CAE calculation.

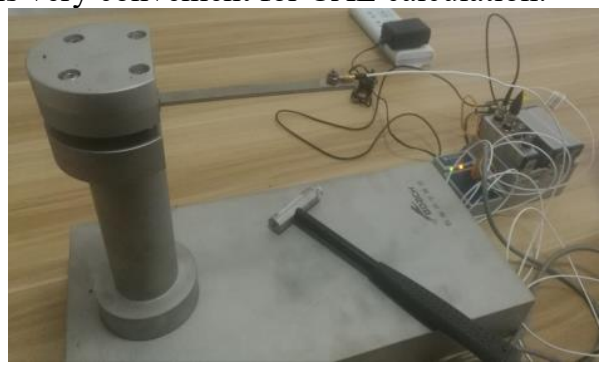

Fig. 2 PVC Film Material Property Measurement Experiment Table

Fig. 2 shows the results of thin films and splines on the experimental platform, and Fig. 3 shows the measured data results.
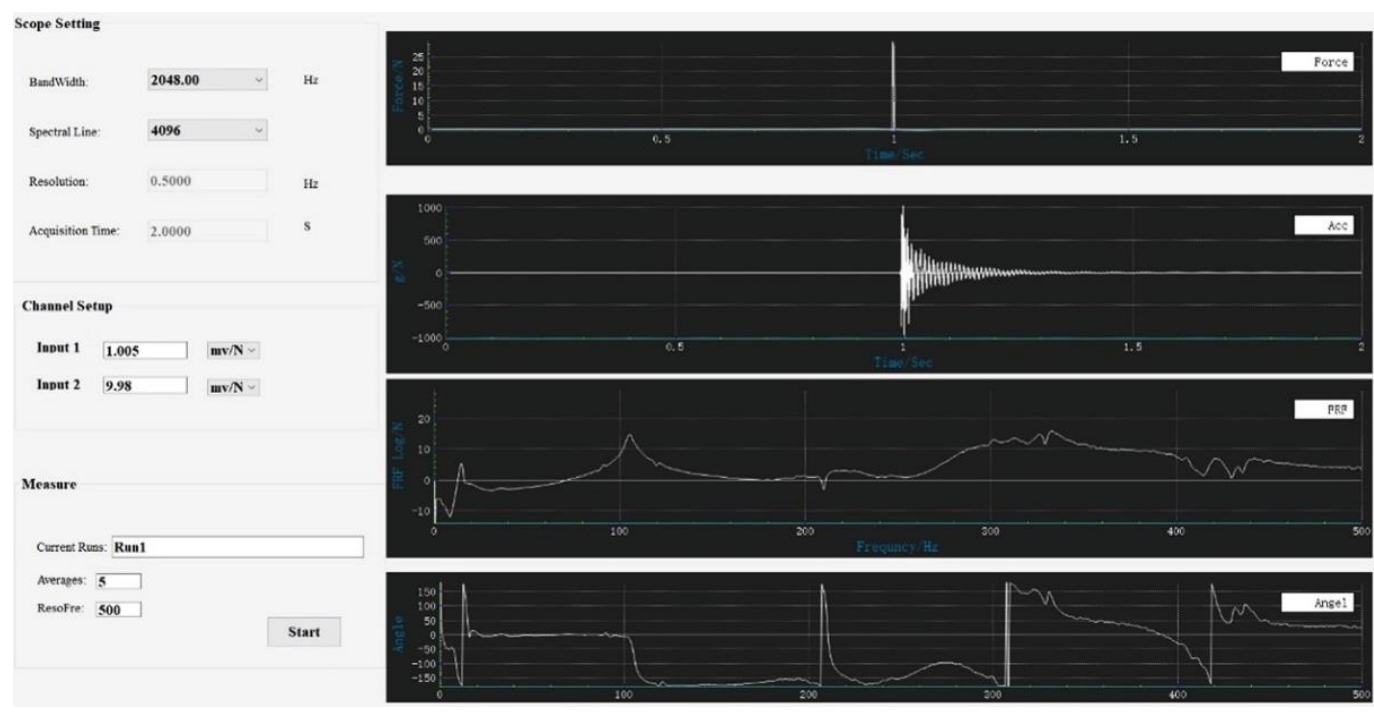

Fig. 3 Acoustic Measure Software

After measurement, the Young's modulus of PVC film material used this time is $3800 \mathrm{Mpa}$, and the loss factor is 0.1 . 


\section{Acoustic Simulation and Experimental Test}

In this paper, the modal method is used to acquire the simulation calculation of sound insulation factor of thin-film acoustic metamaterial, and the parameters used are acquired by the above method. As shown in Fig. 4, the massless acoustic metamaterial is only a thin film. The sound field finite element is established as shown in Fig. 5.

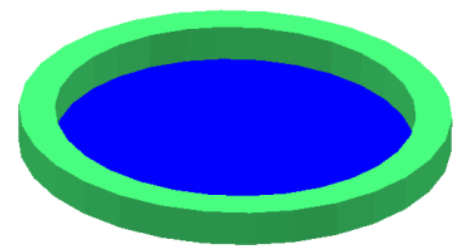

Fig. 4 Simulation Model Diagram of Counter weightless Structure

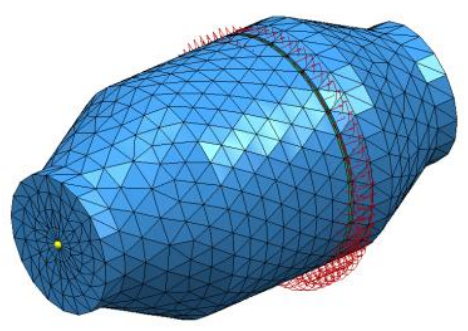

Fig. 5 Simulation Grid Calculation Diagram of Counterweight-free Structure

Fig. 6 shows the results of the film with added counterweight, and Fig. 7 shows the finite element method of the sound field.

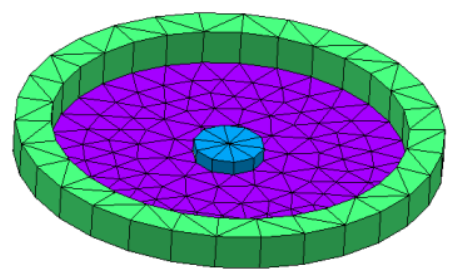

Fig. 6 Simulation Model Diagram of Counterweight Structure

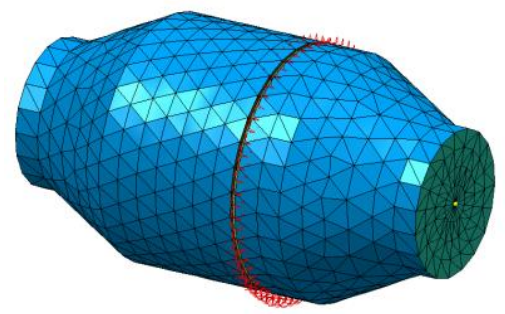

Fig. 7 Calculation Diagram of Counterweight Structure Simulation Grid

\section{Acoustic Experiment and Result Analysis}

In order to acquire the consistency between simulation and experiment, in this paper, the method of direct comparative analysis between fax results and experimental results is adopted. At this experimental platform, the standing wave tube measurement system is used as shown in Fig. 8. The measurement is carried out by means of transmission loss measurement. The sample with counterweight is shown on the right side of Fig. 8. The picture of the sample without counterweight will not be given here. In this experiment, $0.2 \mathrm{~mm}$ PVC film was used. After measurement, it is known that its Young's modulus was $3.8 \times 109 \mathrm{Mpa}$ and its density was $1400 \mathrm{~kg} / \mathrm{m} 3$.The counterweight used is a stainless steel sheet with a mass of $1.22 \mathrm{~g}$, a diameter of $10 \mathrm{~mm}$, and a thickness of $2 \mathrm{~mm}$.

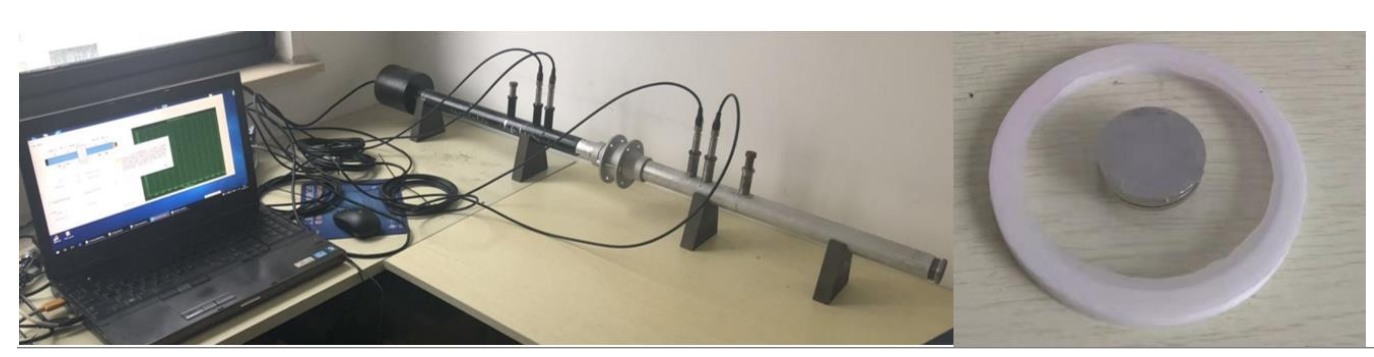

Fig. 8 Acoustic Standing Wave Tube Measuring System

As shown in Fig. 9, the experimental and simulation results are in good consistency, which shows that the thin film materials acquired by the acoustic scheme are accurate and correct, and it is feasible to study acoustic metamaterials by finite element method. 


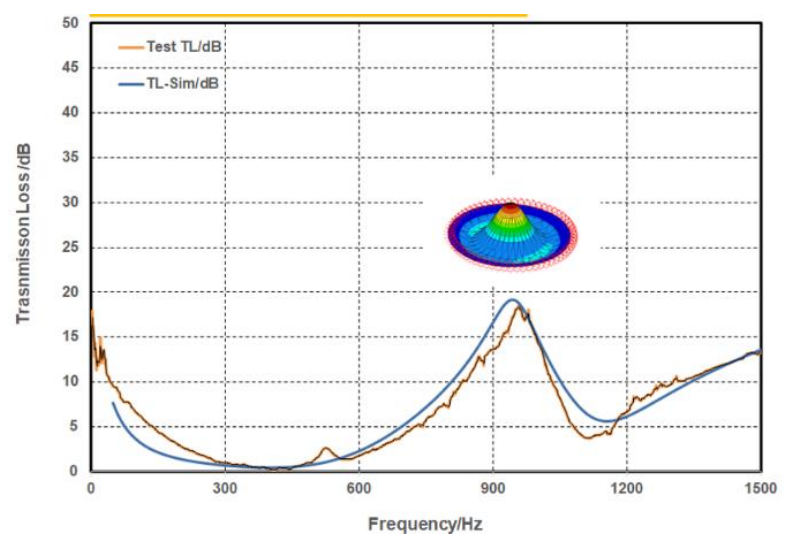

Fig. 9 Result Comparison of Counter weightless Experiment and Simulation

Fig. 10 shows the comparison between simulation and experiment under the condition of counterweight. Through comparison, it can be seen that there is good consistency of the resonance frequency points at both low frequency and high frequency.

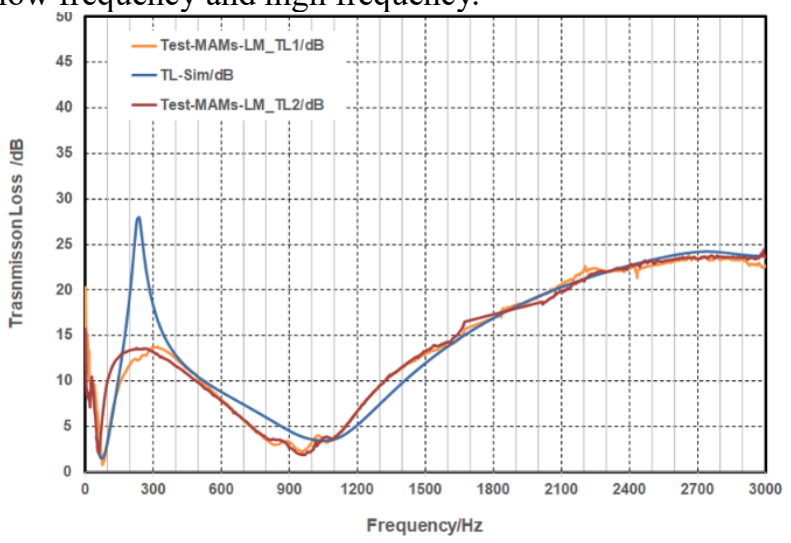

Fig. 10 shows the result comparison between the counterweight experiment and the simulation.

Analysis of Fig. 9 shows that there is no sound insulation effect in the center frequency range of $300 \mathrm{~Hz}$ without counterweight, but after counterweight is added, there is sound insulation effect at the center frequency of $300 \mathrm{~Hz}$, indicating that sound insulation effect is achieved at this time under the interaction of counterweight and membrane.

\section{Conclusion}

The difficulty in practical application of acoustic metamaterials lies in the accuracy of acquiring the parameters of thin film materials. Many examples prove that the difficulty of applying metamaterial theory to practical engineering lies in that. Experiments prove that the scheme of acquiring the parameters of thin film materials by acoustic method is reliable and more conducive to practical engineering application. Under the condition of complex analytical solution, the use of simulation method is feasible and accurate, and has a good impetus to the practical application of acoustic metamaterials.

\section{Acknowledgments}

Project Supported by Science and Technology project of State Grid Corporation of China. (Research on vibration control and noise reduction technology about large oil-immersed transformer)

\section{References}

1. Liu, Z., Zhang, X., Mao, Y., Zhu, Y.Y., Yang, Z., Chan, C.T. , Sheng, P. (2000) Locally Resonant Sonic Materials. Science, 289, 1734-1736.

2. Wu Y., Lai Y., Zhang Z. Q. (2007) Effective medium theory for elastic metamaterials in two dimensions. Physical Review B, 76(20): 205313.

3. Yang Z., Mei J., Yang M., et al. (2008) Membrane-Type Acoustic Metamaterial with Negative Dynamic Mass. Physical Review Letters, 101(20): 204301.

4. Naify C. J., Chang C. M., Mcknight G., et al. (2010) Transmission loss and dynamic response of membrane-type locally resonant acoustic metamaterials. Journal of Applied Physics, 108(11): 204301.

5. Langfeldt F., Riecken J., Gleine W., et al. (2016) A membrane-type acoustic metamaterial with adjustable acoustic properties. Journal of Sound and Vibration, 2016: S0022460X16002728.

6. Zhang Y., Wen J., Xiao Y., et al. (2012) Theoretical investigation of the sound attenuation of membrane-type acoustic metamaterials. Physics Letters A, 376(17): 1489-1494.

7. Lin G. C., Chen S. Q., Li Y. L., et al. (2017) Transmission Loss of Membrane-Type Acoustic Metamaterial with Negative Effective Mass. Materials Science Forum, 898: 1749-1756.

8. Ma F., Wu J. H., Huang M. (2015) Resonant modal group theory of membrane-type acoustical metamaterials for low-frequency sound attenuation. The European Physical Journal Applied Physics, 71(3): 30504.

9. Tian H., Tong D., Tao Y. (2018) Analytical approach of membrane-type acoustic metamaterial with ring masses. Multidiscipline Modeling in Materials and Structures, 2018.

10. Xiao Y., Wen J., Wen X. (2012) Sound transmission loss of metamaterial-based thin plates with multiple subwavelength arrays of attached resonators. Journal of Sound and Vibration, 331(25): 5408---5423.

11. Ma F., Wu J. H., Huang M., et al. (2015) A purely flexible lightweight membrane-type acoustic metamaterial. Journal of Physics D: Applied Physics, 48(17): 175105.

12. Lu K., Wu J. H., Guan D., et al. (2016) A lightweight low-frequency sound insulation membrane-type acoustic metamaterial. AIP Advances, 6(2): 025116. 